\title{
Transporte e ressuspensão de sedimentos finos por ondas sobre um leito viscoelástico
}

\author{
Juliana S. Ziebell, \\ Instituto de Matemática, Estatística e Física, IMEF, FURG, \\ 96201-900, Rio Grande, RS \\ E-mail: julianaziebell@furg.br, jusziebell@gmail.com, \\ Leandro Farina \\ UFRGS - Instituto de Matemática Pura e Aplicada \\ 91509-900, Porto Alege, RS \\ E-mail: farina@mat.ufrgs.br.
}

\section{Resumo:}

Soluções numéricas da equação do transporte unidimensional que descrevem a evolução da concentração de sedimentos suspensos sobre um leito viscoelástico foram obtidas para alguns casos particulares.

Usando o modelo viscoelástico generalizado de [5] para definir a camada de lama viscoelástica, obtivemos uma nova equação do transporte unidimensional para esse mesmo problema.

Palavras-chave: Ondas em fluidos, Lama viscoelástica, Equação do transporte

\section{Introdução}

O movimento dos sedimentos no fundo de um meio aquático, causado pelo fluido, tem sido considerado um importante problema a ser estudado na engenharia costeira e na geologia [4].

O artigo [4] apresentou uma teoria analítica para ondas harmônicas simples sobre um fundo plano, a fim de examinar como ondas transportam sedimentos finos sozinhas, sem o efeito do vento, pela convecção e difusão, depois de ressuspendê-los do fundo. Para obter essa equação o método de múltiplas escalas foi empregado.

Já se uma camada de água está sobre uma camada de lama fluida, seu movimento oscilatório pode ser alterado. Desse modo, o transporte de partículas suspensas na camada limite que fica sobre a interface entre as duas camadas também pode ser modificado, tornando-se um interessante objeto de estudo.

De acordo com [1], o modelo mais adequado para representar as propriedades reológicas da lama fluida é o modelo viscoelástico. Há duas maneiras de definir esse modelo: o corpo de Voight [2] e o modelo viscoelástico generalizado [5].

Neste trabalho, avaliamos o modelo proposto em [8], apresentando resultados numéricos da equação do transporte para a concentração de sedimentos no fundo da água sob um leito viscoelástico definido como um corpo de Voight. Ainda, considerando o modelo viscoelástico generalizado, obtivemos uma nova equação do transporte para a concentração desses sedimentos.

\section{O problema}

Consideramos um sistema de duas camadas. A camada superior é formada por água de profundidade $h$ e está sobre uma fina camada de lama fluida, a qual estamos supondo viscoelástica, de profundidade $d$. Assumimos também que a profundidade da lama fluida é constante. De acordo 
com [5], em águas costeiras a profundidade típica $d$ da lama é geralmente de ordem $0.5 \mathrm{~m}$ e é muito menor do que a profundidade $h$ da camada superior de água, logo, $h>>d$.

Suponhamos que uma onda progressiva de pequena amplitude se propague na direção horizontal $x$ sob a superfície da água, cujo deslocamento é dado por

$$
\eta=\operatorname{Re}\left[a \mathrm{e}^{i(k x-\sigma t)}\right]
$$

onde $a$ é a amplitude da onda, $i$ é a unidade do número complexo, $k$ é o número de onda, $\sigma$ é a frequência angular da onda e $t$ é o tempo. Estamos considerando $a, \sigma \in \mathbb{R}$ e $k \in \mathbb{C}$.

Ainda, consideramos o eixo vertical $z$ apontando verticalmente para cima. Assim, definiremos o deslocamento da interface por

$$
z=\xi(x, t)=b \mathrm{e}^{i(k x-\sigma t)} .
$$

Por fim, denotamos as densidades da água e da lama fluida por $\rho_{w}$ e $\rho_{m}$, respectivamente.

Consideramos ondas de comprimento e amplitude pequenos de modo que $k h=\mathcal{O}(1)$ e $k a<<1$. Vamos estudar o caso quando a espessura da camada de lama fluida é comparável à espessura da camada limite de Stokes.

Além disso, assumimos que a profundidade da lama fluida $d$ e a espessura da camada limite que surge sob a lama fluida $\delta_{m}=\left(2 \nu_{m} / \sigma\right)^{1 / 2}$ são ambas comparáveis com a amplitude da onda sobre a superfície da água $a$, do mesmo modo que foi proposto em [3]. Ainda, a camada limite de Stokes tem espessura dada por $\delta_{w}=\left(2 \nu_{w} / \sigma\right)^{1 / 2}$ e é desenvolvida na interface entre a água e a lama fluida, onde $\nu_{w}$ é a viscosidade da água como em [3].

Definimos a declividade da onda por $\epsilon=k a<<1$, que é escolhido como um parâmetro pequeno.

\section{Modelo 1: Corpo de Voight}

Para obtermos a solução, expandimos as coordenadas da velocidade

$$
\left(u_{f}, v_{f}\right)=\left(u_{f_{0}}, v_{f_{0}}\right)+\epsilon\left(u_{f_{1}}, v_{f_{1}}\right)+\mathcal{O}\left(\epsilon^{2}\right)
$$

e assumimos que as componentes da velocidade de primeira ordem e a onda da interface são definidas pelo mesmo harmônico que a superfície da onda, ou seja,

$$
\left(u_{f_{0}}, v_{f_{0}}, \xi\right)=\operatorname{Re}\left[\left(\tilde{u}_{f}, \tilde{v}_{f}, b\right) \mathrm{e}^{i(k x-\sigma t)}\right]
$$

onde a condição cinemática na interface é

$$
\frac{\partial \xi}{\partial t}=\left.v_{w_{0}}\right|_{z=-h}
$$

As soluções gerais das equações acima foram obtidas em [7] e são dadas por, respectivamente,

$$
\begin{gathered}
\tilde{u}_{w}=\left(1+D \mathrm{e}^{-\lambda_{w} z}\right) \tilde{U}_{I}, \text { em } z>0, \\
\tilde{u}_{m}=\left[\gamma-\gamma \cosh \left(\lambda_{m}(z+d)\right)+H \operatorname{senh}\left(\lambda_{m}(z+d)\right)\right] \tilde{U}_{I}, \text { em } 0>z>-d, \\
\tilde{v}_{w}=-i k \lambda_{w}^{-1}\left(\lambda_{w} z+B-D \mathrm{e}^{-\lambda_{w} z}\right) \tilde{U}_{I} \text { em } z>-h
\end{gathered}
$$

$\mathrm{e}$

$$
\tilde{v}_{m}=-i k \lambda_{w}^{-1}\left[\gamma\left(\lambda_{w}(z+d)-\operatorname{senh}\left(\lambda_{w}(z+d)\right)+H\left(\cosh \left(\lambda_{w}(z+d)\right)-1\right)\right] \tilde{U}_{I}, \text { em }-h>z>-d,\right.
$$

onde

$$
B=\zeta\left[\gamma\left(\lambda_{m} d-\operatorname{senh}\left(\lambda_{m} d\right)\right)+H\left(\cosh \left(\lambda_{m} d\right)-1\right)\right]+D
$$




$$
D=\frac{-\gamma \zeta-(1-\gamma) \zeta \cosh \left(\lambda_{m} d\right)}{\zeta \cosh \left(\lambda_{m} d\right)+\gamma \operatorname{senh}\left(\lambda_{m} d\right)}
$$

$\mathrm{e}$

$$
H=\frac{\gamma(1-\gamma)+\gamma^{2} \cosh \left(\lambda_{m} d\right)+\gamma \zeta \operatorname{senh}\left(\lambda_{m} d\right)}{\zeta \cosh \left(\lambda_{m} d\right)+\gamma \operatorname{senh}\left(\lambda_{m} d\right)}
$$

sendo

$$
\zeta=\left(\nu_{m}^{+} / \nu_{w}\right)^{1 / 2}
$$

um parâmetro complexo relacionado com a variação da viscosidade, onde

$$
\nu_{m}^{+}=\nu_{m}+i G_{m} / \rho_{m} \sigma
$$

é o parâmetro complexo viscoelástico. Observamos que nesse ponto o modelo viscoelástico [8] se diferencia do modelo viscoso [6], pois nesse último $G_{m}=0$.

A amplitude do deslocamento da interface é dada por

$$
b=\left.i \sigma^{-1} \tilde{v}_{w}\right|_{z=-h}=k \sigma^{-1} \lambda_{w}^{-1}(B-D) \tilde{U}_{I}
$$

Após determinarmos a velocidade da água, podemos avaliar como se desenvolve o transporte de sedimentos gerado pelas ondas.

\section{Modelo 2: Modelo viscoelástico generalizado [5]}

Para a camada de água, usamos a seguinte normalização

$$
\begin{array}{r}
\left(x^{\prime}, z^{\prime}, h\right)=\frac{g}{\sigma^{2}}(x, z, H), t^{\prime}=\sigma^{-1} t, \quad k^{\prime}=\frac{\omega^{2}}{g} k, \\
\Phi^{\prime}=\frac{a_{0} g}{\sigma} \Phi, \quad \eta^{\prime}=a_{0} \eta, \quad\left(P^{\prime(w)}, p^{(w)}\right)=\rho^{(w)} g a_{0}\left(P^{(w)}, p^{(w)}\right),
\end{array}
$$

onde $g$ é a gravidade, $\rho^{(w)}$ é a densidade da água, $\sigma$ é a frequência e $t$ é o tempo.

Para a camada de lama fluida, definimos uma nova coordenada vertical medida a partir do fundo dessa camada $Z^{\prime}=z^{\prime}+h+d$ e usamos a seguinte normalização

$$
\begin{array}{r}
x^{\prime}=\frac{g}{\sigma^{2}} x, \quad Z^{\prime}=d Z, t^{\prime}=\sigma^{-1} t, \quad \xi^{\prime}=\epsilon a_{0} \xi \\
u^{\prime}=a_{0} \sigma u, v^{\prime}=\left(\epsilon a_{0} \sigma \frac{d}{a_{0}}\right) v, \quad\left(p^{\prime}, P\right)=\left(\gamma \rho^{(m)} g a_{0}\right)\left(p^{(m)}, P^{(m)}\right) .
\end{array}
$$

\subsection{Solução do problema}

Como em [5], expandimos em série de potências as variáveis $u_{f}, v_{f}, \Phi, \ldots$, e escrevemos cada incógnita $\mathcal{F}$ como

$$
\mathcal{F}=\mathcal{F}_{0}+\epsilon \mathcal{F}_{1}+\epsilon^{2} \mathcal{F}_{2}+\mathcal{O}\left(\epsilon^{3}\right),
$$

onde $\left(\eta_{n}, \xi_{n}\right)$ dependem de $\left(x, x_{1} ; t, t_{1}\right), \Phi_{n}=\Phi_{n}\left(x, x_{1} ; z ; t, t_{1}\right)$ na água e $\left(u_{n}, v_{n}\right)$ de $\left(x, x_{1} ; Z ; t, t_{1}\right)$ na lama fluida.

Ainda, definimos que

$$
\begin{gathered}
\mathcal{F}_{0}=\mathcal{F}_{00}+\left(\mathcal{F}_{01} \mathrm{e}^{i \psi}+\text { c.c. }\right) \\
\mathcal{F}_{1}=\mathcal{F}_{10}+\left(\mathcal{F}_{11} \mathrm{e}^{i \psi}+\text { c.c. }\right)+\left(\mathcal{F}_{21} \mathrm{e}^{2 i \psi}+\text { c.c. }\right) \\
\mathcal{F}_{2}=\mathcal{F}_{20}+\left(\mathcal{F}_{21} \mathrm{e}^{i \psi}+\text { c.c. }\right)+\left(\mathcal{F}_{22} \mathrm{e}^{2 i \psi}+\text { c.c. }\right)+\left(\mathcal{F}_{32} \mathrm{e}^{3 i \psi}+\text { c.c. }\right)
\end{gathered}
$$

e assim sucessivamente, onde $\mathcal{F}_{n m}=\mathcal{F}_{n m}\left(z, x_{1}, t_{1}\right) \forall n, m \in \mathbb{N}$. Observemos que assim temos $\mathcal{F}_{n m, x}\left(z, x_{1}, t_{1}\right)=\mathcal{F}_{n m, t}\left(z, x_{1}, t_{1}\right)=0 \forall n, m \in \mathbb{N}$. 
Com essas hipóteses, [5] mostrou que as coordenadas da velocidade de $\mathcal{O}(1)$ e $\mathcal{O}(\epsilon)$ são dadas por

$$
\begin{gathered}
u_{w_{01}}=\frac{\partial}{\partial x}\left(\Phi_{01} \mathrm{e}^{i \psi}\right)=i k_{0} \Phi_{01} a_{0} \sigma=\frac{k_{0} A}{2} \frac{\cosh Q}{\cosh q} \\
v_{w_{01}}=-\frac{i A k_{0}}{2} \frac{\operatorname{senh} Q}{\cosh q}, \\
u_{w_{10}}=\frac{\partial \Phi_{00}}{\partial x_{1}}, \\
u_{w_{11}}=\left[\frac{-i}{2} \frac{\partial A}{\partial x_{1}} \frac{\cosh Q}{\cosh q}+i k_{0}\left(i k_{1} C_{g} A \operatorname{senh} q \operatorname{senh} Q-\frac{Q \operatorname{senh} Q}{2 k_{0} \cosh q} \frac{\partial A}{\partial x_{1}}\right)\right], \\
u_{w_{12}}=i k_{0} \frac{3 i}{8} \frac{A^{2}}{\operatorname{senh}^{4} q} \cosh (2 Q), \\
v_{w_{10}}=0, \\
v_{w_{10}}=\left[i k_{1} k_{0} C_{g} A \operatorname{senh} q \cosh Q-k_{0} \frac{\operatorname{senh} Q+Q \cosh Q}{2 k_{0} \cosh q} \frac{\partial A}{\partial x_{1}}\right]
\end{gathered}
$$

$\mathrm{e}$

$$
v_{w_{12}}=\frac{3 i k_{0}}{8} \frac{A^{2}}{\operatorname{senh}^{4} q} \operatorname{senh}(2 Q)
$$

\section{Método}

Por [8] e [4], a equação do transporte de massa pode ser escrita como

$$
\frac{\partial C}{\partial t}+\epsilon u_{w} \frac{\partial C}{\partial x}+\left(\epsilon v_{w}-v_{f}\right) \frac{\partial C}{\partial z}=\epsilon^{2} E \frac{\partial^{2} C}{\partial x^{2}}+E \frac{\partial^{2} C}{\partial z^{2}} .
$$

A equação (32) está sujeita às condições de contorno

$$
\begin{gathered}
v_{f} C+E \frac{\partial C}{\partial z}=\epsilon^{2} \mathcal{E}\left|\tau_{b}\right|, \text { em } z=\epsilon \xi . \\
C=0 \text { in } z \rightarrow \infty .
\end{gathered}
$$

Como Ng e Wu [8], expandimos a concentração e as derivadas temporais como

$$
C \rightarrow C_{0}+\epsilon C_{1}+\epsilon C_{2}+\mathcal{O}\left(\epsilon^{3}\right)
$$

e

$$
\frac{\partial}{\partial t} \rightarrow \frac{\partial}{\partial t}+\epsilon^{2} \frac{\partial}{\partial T}
$$

onde $T=\epsilon^{2} t$ é o tempo lento para descrever os processos de transportes longitudinais.

Esperamos que o termo de ordem zero $C_{0}=C_{0}(x, z, T)$ represente a concentração principal no tempo e portanto não depende do tempo rápido $t$, enquanto que os termos de ordem mais alta $C_{n}=C_{n}(x, z, t, T)$, para $n=1,2, \ldots$ são funções de ambos os tempos.

A solução da da equação da concentração de sedimentos é obtida através das equações de ordem $\mathcal{O}(1), \mathcal{O}(\epsilon)$ e $\mathcal{O}\left(\epsilon^{2}\right)$. Essa solução depende das coordenadas da velocidade da água, que por sua vez, dependem de como a camada de lama fluida é definida. 


\subsection{Concentração de sedimentos no fundo - modelo 1}

A equação do transporte para a concentração de partículas no fundo foi obtida em [8] e é dada por

$$
\frac{\partial C_{b}}{\partial T}+U \frac{\partial C_{b}}{\partial x}=\left[E-D_{T}\right] \frac{\partial^{2} C_{b}}{\partial x^{2}}
$$

onde

$$
U=\frac{\left\langle\tilde{u}_{w_{2}} F\right\rangle}{\langle F\rangle}+\frac{\operatorname{Re}\left[\left\langle\tilde{u}_{w}^{*} M\right\rangle-\left.b \tilde{u}_{w}^{*}\right|_{z=-h}\right]}{2\langle F\rangle} \text { e } D_{T}=\frac{\operatorname{Re}\left\langle\tilde{u}_{w}^{*} N\right\rangle}{2\langle F\rangle} .
$$

As funções $M$ e $N$ dependem de $z$ e são dadas por

$$
N(z)=\sigma^{-1} \tilde{U}_{I}\left(A_{1} e^{-\beta z / \alpha}-i e^{-z / \alpha}+A_{2} e^{-\left(A_{0}+1\right) z / \alpha}\right)
$$

$\mathrm{e}$

$$
M(z)=k \sigma^{-1} \tilde{U}_{I}\left[A_{3} e^{-\beta z / \alpha}+A_{4} e^{-z / \alpha}+A_{5} e^{-\left(A_{0}+1\right) z / \alpha}+(z / \alpha) e^{-z / \alpha}\right]
$$

onde $A_{i}$ são constantes para $i=\{1,2,3,4,5\}$.

\subsubsection{Resultados numéricos}

Consideramos os seguintes valores para os parâmetros normalizados:

i) $\gamma=0.8, \hat{\delta}_{m}=5$ a $8, \hat{\lambda}_{e}=0.3,0.15$ e 0.1

ii) $\hat{\lambda}_{m}=\mathcal{O}(0.1), \hat{d}=\mathcal{O}(10), P e=S c=1$.

As soluções numéricas que apresentaremos nesse trabalho foram obtidas usando a rotina desenvolvida em Fenics project [9]. Para uma concentração inicial $C_{b}(x, 0)=100$ obtivemos o gráfico apresentado na figura 1.

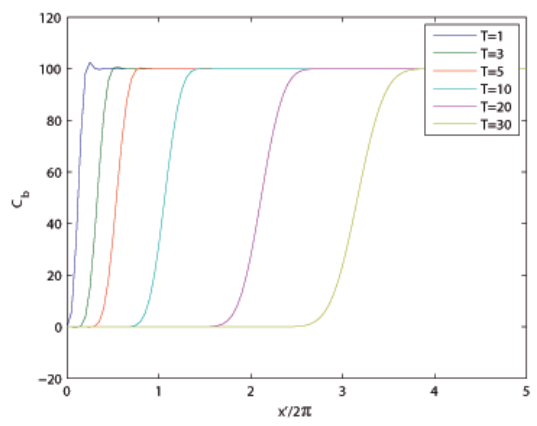

Figura 1: $C_{b}$ como função de $x / 2 \pi$ para $C_{b}(x, 0)=100$ e $T=1,3,5,10,20$, e 30 .

A seguir consideramos que há erosão em uma faixa para $x^{\prime} / 2 \pi \in[0,1]$ como em [4]. Usando a mesma normalização, a equação que formula o problema será dada por

$$
\frac{\partial C^{\prime}}{\partial T^{\prime}}+\hat{U} \frac{\partial C^{\prime}}{\partial x^{\prime}}=\left[\hat{E}-\hat{D_{T}}\right] \frac{\partial^{2} C^{\prime}}{\partial x^{\prime 2}}+\mathcal{E}_{\mathcal{R}}^{\prime}\left(x^{\prime}\right)
$$

onde

$$
\mathcal{E}_{\mathcal{R}}{ }^{\prime}\left(x^{\prime}\right)= \begin{cases}1, & 0<x^{\prime}<L^{\prime}=k L \\ 0, & x^{\prime}<0, x^{\prime}>L .\end{cases}
$$

Consideramos a concentração inicial nula. Adotamos a mesma normalização usada em [8] e em [4].

A solução da equação do transporte uni-dimensional em um sistema sem a camada de lama fluida e com erosão foi encontrada analiticamente em [4]. Comparamos a solução numérica 


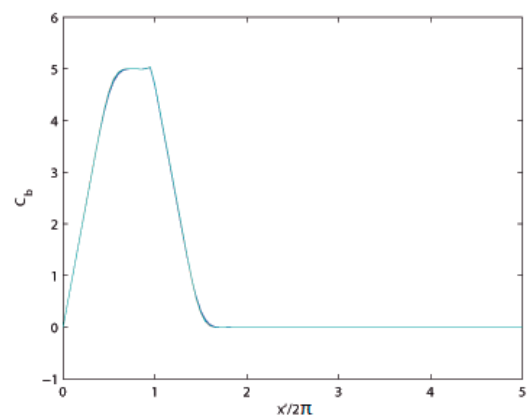

Figura 2: $C_{b}$ como função de $x / 2 \pi$ para $\hat{\delta}_{m}=5, \hat{\lambda}_{m}=0.1$ e $d=0.0,0.25,0.5$ e 1.0 .

obtida por nós e a solução analítica obtida por Mei e vimos que o erro é da ordem de $\mathcal{O}\left(10^{-3}\right)$. A seguir resolvemos a equação para $\hat{d}=\{0.25,0.5,1.0\}$ com $T=5$. O gráfico está na figura 2 . Observemos que não houve mudança para $\hat{d}$ pequeno, já que as curvas estão sobrepostas.

O resultado para $\delta=5, \lambda=0.1$ e $\hat{d}=7,8,9,10,11$ e 12 são dados na figura 3 . Observamos que os picos não sofrem alterações consideráveis ao variar a espessura na camada de lama fluida. Porém, conforme essa camada aumenta, o pico da concentração se desloca para $x^{\prime}$ menor.
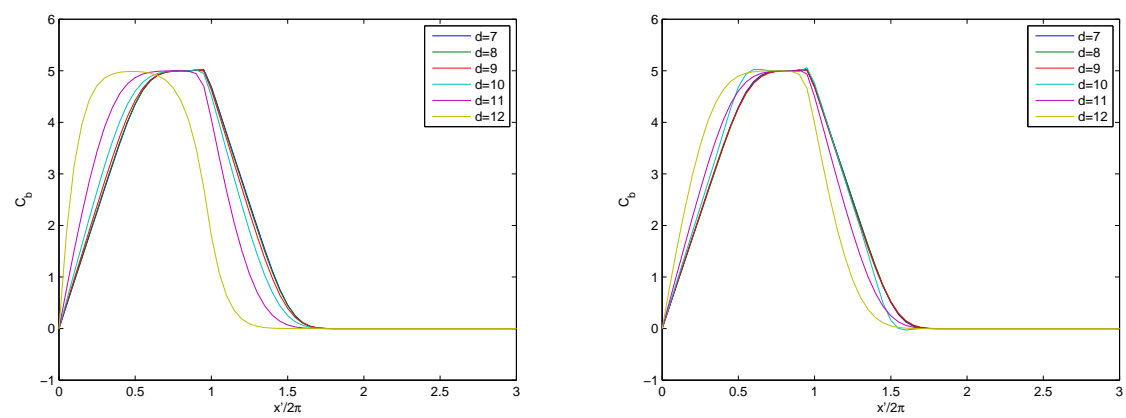

Figura 3: $C_{b}$ como função de $x / 2 \pi$ para $\hat{\delta}_{m}=5$ e $\hat{\delta}_{m}=8, \hat{\lambda}_{m}=0.1$ e $d=7,8,9,10,11$ e 12 e $T=5$.

\subsection{Concentração de sedimentos no fundo - modelo 2}

Obtivemos que a equação adimensional para a concentração no fundo é dada por

$$
\frac{\partial C_{b}}{\partial T}+U \frac{\partial C_{b}}{\partial x}=\left[E-D_{T}\right] \frac{\partial^{2} C_{b}}{\partial x^{2}},
$$

onde

$$
U=\frac{a_{0} \sigma^{2}}{g}\left[\frac{\left\langle\overline{u_{w_{1}}} F\right\rangle}{2\langle F\rangle}+\frac{\operatorname{Re}\left\langle\overline{u_{w_{0}}^{*} M}\right\rangle}{2\langle F\rangle}-\frac{1}{2} \frac{\operatorname{Re}\left(\xi_{01} u_{01}^{*}\right)_{z=0}}{\langle F\rangle}\right] \text { e } D_{T}=-\frac{a_{0} \sigma^{2}}{g} \frac{\operatorname{Re}\left\langle u_{w_{0}}^{*} N\right\rangle}{2\langle F\rangle},
$$

Com esse modelo, as funções $M$ e $N$ são dadas por

$$
M(z)=B_{1} \mathrm{e}^{-\theta z / \alpha}+\left(B_{2} \cosh (\sigma z)+B_{3} \operatorname{senh}(\sigma z)\right) \mathrm{e}^{-z / \alpha}
$$

e

$$
N(z)=A_{1} e^{-\beta z / \alpha}+\left[A_{2} \cosh \left(k_{0} z\right)+A_{3} \operatorname{senh}\left(k_{0} z\right)\right] e^{-z / \alpha},
$$

onde $A_{i}$ e $B_{i}$ são constantes para $i=\{1,2,3\}$. 
Observemos que os coeficientes de dispersão e convecção $D_{T}$ e $U$, respectivamente, têm as mesmas características dos coeficientes obtidos por [8], descritos em (38). O que torna a equação diferente são as coordenadas da velocidade da água de $\mathcal{O}(1)$ e $\mathcal{O}(\epsilon)$ e a onda da interface de $\mathcal{O}(1)$, que foram obtidas com o modelo viscoelástico generalizado. A importância desse resultado se deve ao fato desse modelo ter sido obtido através de um modelo mais realístico visto que ele leva em consideração a dependência dos coeficientes da tensão e deformação da frequência.

\section{Conclusão}

O problema de propagação de ondas de água sobre uma camada de lama fluida viscoelástica foi investigado e a equação que descreve a evolução da concentração de sedimentos, obtida por [8], foi resolvida numericamente para fundos erodíveis e não erodíveis. Comparamos estes resultados com aqueles onde o fundo é rígido [4], unidimensional e com erosão restrita a uma faixa do fundo.

Usando um modelo viscoelástico generalizado para um sistema de duas camadas, obtivemos uma nova equação para a descrição da concentração de sedimentos (43).

Como trabalhos futuros, iremos investigar numericamente a solução dessa nova equação.

\section{$7 \quad$ Agradecimentos}

Trabalho parcialmente financiado pelo CNPq e CAPES,

J. S. Z. agradece ao BCAM (Basque Center for Applied Mathemathics) pela hospitalidade e pelo suporte financeiro parcial. Agradece também ao Dr. Johan Jansson pelo auxílio no trabalho computacional.

\section{Referências}

[1] P. J. De Wit, Liquefaction of cohesive sediments caused by waves, master thesis, Delft University of Technology, vol. 174, Netherlands, 1995.

[2] J. P. Y. Maa and A. J. Mehta, Soft mud properties: voight model, J. Waterway, vol. 114, 765-770, 1988.

[3] C.C. Mei and C. Chian, Dispersion of small suspended particles in a wave boundary layer, American Meteorological Society, 2479-2495, 1994.

[4] C. C. Mei, She-jun Fan and Kang-ren Jin, Resuspension and transport of fine sediments by waves, J. Geophysical Research, vol. 102, 15.807-15.821, 1997.

[5] C. C. Mei, M. Krotov, and Z. Huang, A. Huhe, Short and long waves over a muddy seabed, J. Fluid. Mech., vol. 643, 33-58, 2010.

[6] C. O Ng., Water waves over a muddy bed: a two layer Stokes boundary layer model, Coastal Engineering, vol. 40, 221-242, 2000.

[7] C. O. Ng and X. Zhang, Mass transport in water waves over a thin layer of soft viscoelastic mud, J. Fluid. Mech., vol. 573, 105-130, 2007.

[8] C. O. Ng and C. H. Wu, Dispersion of suspended particles in a wave boundary layer over a viscoelastic bed, International Journal of Engineering Science, vol. 46, 50-65, 2008.

[9] M. Schläger and Fenics Team, Fenics Project, http://fenicsproject.org/, 2013. cited: June, 05, 2013. 